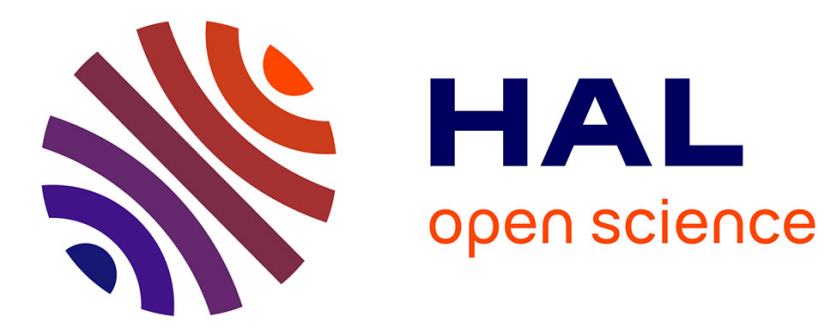

\title{
Retraite et dévotion dans les romans monastiques de Michel-Ange Marin
}

Nicolas Brucker

\section{To cite this version:}

Nicolas Brucker. Retraite et dévotion dans les romans monastiques de Michel-Ange Marin. Dix-

Huitième Siècle, 2016, Se retirer du monde, 48, pp.73-88. 10.3917/dhs.048.0073 . hal-01453499

\author{
HAL Id: hal-01453499 \\ https://hal.science/hal-01453499
}

Submitted on 2 Feb 2017

HAL is a multi-disciplinary open access archive for the deposit and dissemination of scientific research documents, whether they are published or not. The documents may come from teaching and research institutions in France or abroad, or from public or private research centers.
L'archive ouverte pluridisciplinaire HAL, est destinée au dépôt et à la diffusion de documents scientifiques de niveau recherche, publiés ou non, émanant des établissements d'enseignement et de recherche français ou étrangers, des laboratoires publics ou privés. 


\section{RETRAITE ET DÉVOTION DANS LES ROMANS MONASTIQUES DE MICHEL-ANGE MARIN}

Le couvent féminin est, dans le roman du $18^{\mathrm{e}}$ siècle, habituellement dépeint comme un lieu d'enfermement, où des jeunes filles, arrachées à leurs familles, sont retenues contre leur gré, et sacrifiées à des intérêts tout matériels, que la religion pare d'un voile mystique. Le père Michel-Ange Marin, en choisissant de représenter la vie conventuelle sous des couleurs sinon attrayantes, du moins acceptables, entend faire pièce à un préjugé universellement répandu. Ce religieux minime, prédicateur talentueux, directeur, confesseur, gardien, fondateur d'une communauté séculière, les Sœurs de la Retraite, si l'on en croit la notice qui lui a été consacrée ${ }^{1}$, connaît, de l'intérieur, l'univers monastique féminin. Lui qui doit à sa famille spirituelle son attachement à la vie retirée, a fait du thème de la retraite un élément central de ses fictions ${ }^{2}$, et un marqueur de l'opposition entre l'horizon sécularisé des Lumières et la pensée chrétienne orthodoxe. On comprend que Chateaubriand lui ait fait une place, entre l'abbé Gérard et Jean-Pierre Camus, dans son Panthéon des auteurs chrétiens ${ }^{3}$.

\section{Le théâtre des vertus}

Dans Adélaïde de Witsbury, on peut lire cette histoire insérée qui met en scène une dame de Valence, qui « avait le défaut de mal parler de tout le monde », mais avec un esprit tel qu'on l'écoutait avec intérêt, jusqu'au jour où les victimes de sa médisance se liguèrent contre elle, et l'isolèrent si bien qu'elle se trouva réduite

\footnotetext{
${ }^{1}$ Dom Mayeul Chaudon (abbé), Éloge historique du R.P. Michel-Ange Marin, Mercure de France, juillet 1767, p. 64-71; reproduit en tête des Lettres ascétiques, Niel, Avignon, 1769.

${ }^{2}$ On trouvera un résumé de chacun des neuf romans en annexe de mon article, "Voies féminines de la conversion dans les romans du Père Michel-Ange Marin », dans La conversion. Expérience spirituelle, expression littéraire, dir. Nicolas Brucker, Berne, Peter Lang, 2005, p. 119-140.

${ }^{3}$ Chateaubriand, Défense du Génie du cbristianisme, éd. Maurice Regard, «Bibliothèque de la Pléiade », Paris, Gallimard, 1978, p. 1104.
} 
au silence. Elle en mourut de dépit ${ }^{4}$. Le monde c'est le salon de Célimène, théâtre de la parole trompeuse et venimeuse. En se retirant de la scène, en privant la médisante de son public, les dames ont infligé à celle-ci la seule punition possible : isolée, elle s'évanouit. Les artifices de la vie mondaine, que Marin condamne à la suite de toute une tradition augustinienne, ne prospèrent que par la complicité de ceux qui s'y complaisent par lâcheté ou par désœuvrement. Par un penchant naturel au mal, les hommes réunis en société favorisent la contagion du vice. Le vrai chrétien, selon Marin, sait qu'il n'est de solution que dans la fuite, le retrait et l'isolement volontaires. Mais alors la vie chrétienne serait-elle un anti-théâtre ? N'y aurait-il rien à montrer de la vertu ? L'homme de bien sera-t-il toujours raillé ? Et Célimène, malgré son vice, toujours désirable? Le roman chrétien du $18^{e}$ siècle, sous les auspices de Jean-Pierre Camus, relève le défi de rendre la vertu aimable, et son imitation désirable. Marin va plus loin dans cette voie, puisque la vertu qu'il met en scène se trouve confinée dans les murs d'un monastère ou d'un ermitage. En désertant la scène du monde, l'auteur nous fait signe de le suivre sur une autre scène ; il lève l'épais rideau de la clôture, nous entraîne là où nul n'a accès, dans les espaces les plus reculés, les plus secrets, jusque dans le repli des consciences et les méandres de l'intériorité. Il anticipe de vingt ans la peinture que Diderot fera du couvent, avec une intention certes antagoniste, mais en recourant à des moyens littéraires, topoï descriptifs et choix énonciatifs, assez similaires. L'épisode de la vêture en est un exemple probant: ni Marin ni Diderot n'en donnent une représentation littérale. Le premier l'élide pour souligner la réaction de la mère de la professante, raille sa sensibilité hors de saison, et désamorce la charge émotionnelle que pourrait contenir la scène (An., I, p. 102-105); le second le réduit, selon le processus psychique de refoulement, à une simple évocation, reconstituée sur la base du témoignage des assistants ${ }^{5}$. La Religieuse marche dans les traces du roman monastique de

\footnotetext{
${ }^{4}$ Marin, Adélaïde de Witsbury, ou la pieuse pensionnaire, Avignon, Giroud, 1750 [1743], p. 114116. Désormais abrégé en $A d$.

${ }^{5}$ Diderot, La Religieuse, dans CEuvres, t. II, éd. Laurent Versini, «Bouquins », Paris, R. Laffont, 1994, p. 300.
} 
Marin, et par bien des aspects peut se lire comme une réplique indirecte à celui-ci.

La donnée théâtrale occupe dans l'apprentissage de la vie retirée une place non négligeable. Les attitudes, gestes, paroles qui font la parfaite religieuse, s'apprennent par une longue imitation de la vie des saints. Ceux-ci sont «les modèles que Dieu a donnés pour toutes les âmes pures et religieuses ", lit-on dans l'Imitation. Les pensionnaires de Marin jouent des scènes de la Bible, moyen de «les rendre plus sensibles et de les graver plus profondément dans l'esprit» (Ad., p. 33-34); elles lisent ensemble des Vies des pères des déserts d'orient', telles que celles de saint Onuphre ou de sainte Euphrasie, "si propre à inspirer de l'amour pour la virginité $^{8} »$. Elles en tirent des saynètes. Ainsi de la visite d'Antoine à Paul ermite que Virginie et Rosalie jouent exactement ( $V$., I, p. 52-53). Mais le modèle adorable entre tous c'est Jésus-Christ. Le retraitant doit s'imprégner des évangiles, et singulièrement des épisodes de la vie du Christ qui le montrent en solitude. Le père Crasset, dans Le Chrétien en solitude, en distinguent quatre: les quarante jours au désert, la transfiguration sur le mont Thabor, la nuit au mont des Oliviers, les apparitions du Ressuscité en Galilée?.

De façon plus générale, le modèle est omniprésent. Les figures d'autorité abondent, à commencer par celle de la maîtresse. C'est souvent une tante qui tient ce rôle, personnage proche par la parenté, mais prestigieux par la réputation de sainteté qui l'entoure. Ses avis sont reçus comme des arrêts, ses instructions sont riches d'une longue expérience dans la direction des âmes. Elle-même dit avoir reçu ce qu'elle transmet de telle supérieure vénérable. Marin signifie ainsi l'importance de la tradition : une sagesse se transmet depuis les origines du monachisme. Le théâtre des vertus n'est pas

\footnotetext{
${ }^{6}$ Imitation de Jésus-Christ, I, 18, 4, trad. De Beüil, Paris, Desprez, 1728, p. 87.

7 «A ne considérer que les quarante premières années du siècle, les productions consacrées aux anciens anachorètes ne se comptent pas » (Pierre Naudin, L'expérience et le sentiment de la solitude dans la littérature française de l'aube des Lumières à la Révolution, Paris, Klincksieck, 1995, p. 24). - Marin est lui-même l'auteur de Vies des pères des déserts d'orient (Avignon, Vve Niel, 1761-1764).

${ }^{8}$ Marin, Virginie, ou la Vierge chrétienne, Avignon, Offray, 1817 [1752], I, p. 207. Désormais abrégé en $V$.

${ }_{9}$ Jean Crasset, Le Chrétien en solitude, Paris, Delespine, 1705 [1674]. «Instructions pour ceux qui vont en retraite », II, n. p.
} 
autonome ; un fil invisible relie ses acteurs à une série de héros et d'hérö̈nes, jusqu'à Jésus-Christ, le modèle ultime. En se plaçant dans la lignée des saints, le retraitant prête sa voix et son corps aux modèles dont il entend s'inspirer; il se laisse agir de l'intérieur par un principe qui lui vient du dehors. Pour cela il lui faut se dépersonnaliser, ce qui en langage chrétien s'exprime en termes de renoncement ou de mort à soi-même. Le paradoxe du chrétien vaut bien celui du comédien : s'il veut exister pour Dieu, il doit s'annuler pour les hommes. L'auteur de l'Imitation de Jésus-Christ le dit autrement: «Si vous voulez demeurer ferme et avancer dans la piété, vivez sur la terre comme étranger, et comme banni de votre pays ${ }^{10} »$.

\section{Topographie et fonction de la retraite conventuelle}

La retraite en tant qu'éloignement du monde est étroitement liée à la conversion. En se tournant vers Dieu, le sujet se détourne des réalités terrestres. Le commandement de Jésus - «Si quelqu'un veut venir après moi, qu'il renonce à soi-même, qu'il se charge de sa croix, et qu'il me suive » (Mt 16, 24) - est sans équivoque : la vie chrétienne se vit dans le retrait et l'austérité. Marin, dans ses romans, ne se fait pas faute de l'expliquer; et il le fait de deux manières: par la vie des personnages éponymes et par les instructions dispensées par leurs maitres. La mention et la description des lieux ne sont pas négligées. La thématisation de la retraite, son inscription dans les coordonnées spatiales et temporelles répondent aux exigences du genre romanesque. On distinguera les lieux extérieurs des lieux intérieurs.

Le désert des pensionnaires de Marin est une nature domestiquée. Le jardin où Virginie et Rosalie se retirent, les dimanches et les jours de fête, clos de murailles, se trouve à peu de distance de la ville. Il offre à leur zèle toutes les commodités sans en laisser voir les inconvénients. Précisément décrit, il comprend une vigne, un potager, un fruitier, une campagne agréable, un bosquet de pins et de chênes, un petit bois, un rocher, une double caverne naturelle et une cascade. Sa situation est, nous est-il indiqué, semblable aux déserts des saintes solitaires de la Thébaïde,

${ }^{10}$ Imitation de Jésus-Christ, I, 17, 1, ouvr. cité, p. 82. 
«dont elles lisaient les vies avec dévotion»( $V$., I, p. 51). La chambre est dans l'espace de la maison ou du couvent un lieu où la pensionnaire se retire, afin de se retrouver seule, et se livrer aux exercices de la piété, la lecture, la méditation, l'oraison. L'expression «se retirer dans sa chambre » revient souvent: elle tend à instaurer une alternance entre solitude et communauté. De fait, la vie monastique est rythmée par cette alternance, de sorte que la religieuse n'a le temps de se lasser ni d'une situation ni de l'autre. La retraite spirituelle fait exception : pendant une période donnée, le fidèle vit dans une réclusion presque absolue, entrecoupée des seuls entretiens avec son directeur.

Le mot retraite pourrait suggérer la sédentarité, l'immobilité, voire le repos ${ }^{11}$. Ici il n'en est rien. Mère Scholastique ne cesse de mettre en garde ses protégées contre les pièges de l'illusion. Les faux dévots se plaisent aux lieux retirés ; ils les font servir à leurs vices. Le jardin devient le lieu du mol abandon, la chambre celui de la rêverie heureuse. Ils y trouvent un confort et des commodités étrangers au véritable esprit de la religion. L'évangile est sur ce point sans ambiguïté : «Les renards ont des tanières, et les oiseaux du ciel ont des nids; mais le Fils de l'homme n'a pas où reposer sa tête » (Mt 8, 20). La vie retirée est en réalité soumise aux plus violentes tribulations. C'est pourquoi rien dans l'ameublement de la cellule ne doit tromper sur sa destination. Dans sa chambre, placée au plus haut étage de la maison, façon de signifier le plus grand éloignement de la rue, donc la séparation du monde, Virginie n'a conservé que le strict nécessaire : une table d'un bois grossier, des chaises garnies de jonc, un lit pauvre et étroit, quelques images de papier, aucun miroir apparent. Sa « capucine », comme elle la nomme, en référence à la pauvreté franciscaine, est la demeure de Dieu ( $V$., II, p. 165-166). «Dieu est chez vous quand vous êtes chez lui», écrit le père Crasset ${ }^{12}$. Aussi la jeune fille lui voue-t-elle un saint respect; elle va jusqu'à en baiser les murailles.

\footnotetext{
11 Robert Mauzi, L'Idée du bonbeur dans la littérature et la pensée française au XVIIIe siècle, Paris, Armand Colin, 1965, p. 330-385.

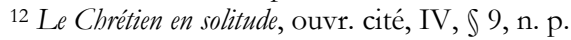


Les espaces retirés ont cette propriété d'être réversibles : la chambre, lieu des révélations, est aussi lieu des tentations. Un jour Virginie est assaillie par des amies qui opposent à son choix de vie les objections du monde; le lendemain elle se rend coupable de distractions, qui lui suggèrent de se prévaloir des progrès qu'elle fait dans la voie spirituelle, et d'en tirer orgueil ( $V$., I, p. 30 et suiv.). Il ne suffit pas d'en être retiré pour s'abstenir du monde. On peut être au cloître et être au monde, soit en esprit, soit par les visites de personnes séculières, soit encore par les nouvelles qu'on prend du dehors, et les entretiens frivoles auxquels elles donnent lieu. L'imagination, source de possibles « évagations ", "sotte bête, qui nous fait galoper par monts et par vaux ${ }^{13} »$, doit être constamment tenue en bride. La dévotion est un combat incessant, une lutte à mort entre les deux parties de l'individu, l'esprit et la chair, selon le dualisme paulinien. La mortification doit aider l'âme à s'affranchir peu à peu de l'emprise du corps, jusqu'à lui imposer une complète domination.

Entre le dehors et le dedans, il est un espace intermédiaire, espace du seuil par lequel peut advenir la mortelle contagion. Le parloir est l'objet d'un discours de mise en garde récurrent. "C'est plus ordinairement par le parloir [...] que le relâchement s'introduit dans les monastères, prévient Angélique; on s'y apprivoise avec le dehors, et on se dégoûte du dedans : on prend l'esprit du monde, et on n'a plus celui de son état $[\ldots] »$ (An., II, p. 11). Mais le parloir peut, exceptionnellement, être le lieu par où s'opère la conversion du monde (Ad., p. 92-93).

Le genre du roman profane, dont Marin imite la façon, rend impératif la référence à des lieux. Une fois installés dans la fiction, ces lieux sont cependant soumis à un processus de dévaluation et de dématérialisation. En montrant qu'il ne suffit pas d'un lieu isolé pour garantir la retraite ; en montrant à l'inverse que l'esprit de retraite peut se vivre indifféremment en tous lieux, y compris au cœur de la société des hommes; en montrant enfin que par l'usage de la méditation le chrétien peut produire en lui des affections auxquelles son corps n'est pas soumis, Marin relativise la notion de

13 Marin, Angélique, ou la religieuse selon le cour de Dieu, Marseille, Mossy, 1828 [1766], II, p. 239. Désormais abrégé en $A n$. 
$\mathrm{lieu}^{14}$. La lecture du roman suit en le reproduisant l'effort que consent la jeune fille pour s'abstraire du monde. Le lecteur est invité lui aussi à renoncer à l'usage qu'il fait habituellement de la fiction, à convertir la lecture de pur divertissement en une lecture à visée didactique, morale et spirituelle. Or celle-ci suppose la prise en compte du symbole, du type et de la figure. Il est aidé dans cet effort par la forme biographique du récit, par son caractère exemplaire et cependant vraisemblable. Le personnage, dont la vie est à la fois ordinaire et singulière, exerce sur lui un pouvoir d'attraction: son éloignement du siècle, son retrait dans la vie immatérielle de l'esprit, son goût croissant pour la solitude appellent chez celui qui en est le spectateur un mouvement analogue.

\section{Une retraite choisie}

Se situant dans la postérité de François de Sales, qui recommandait d' "accommoder la pratique de la dévotion aux forces, aux affaires et aux devoirs de chaque particulier ${ }^{15} »$, Marin indique que la vraie dévotion «consiste à accomplir la volonté de Dieu, chacun dans l'état où la providence l'a placé » ( $V$., I, p. 64). Aussi ses romans définissent-ils des voies singulières pour chacun des personnages, relativement à son milieu, ses capacités, son caractère. La retraite est le temps laissé au sujet pour qu'il puisse discerner quelle voie il devra suivre pour atteindre la perfection chrétienne. Le personnel, exclusivement féminin, que Marin met en scène, se divise en catégories : la vierge, l'épouse, la mère de famille, la veuve, la mondaine ${ }^{16}$. Chaque espèce peut entrer dans la vie dévote, dans l'éloignement du monde et la conformité à l'évangile. Chacune peut tracer son propre chemin de sainteté.

\footnotetext{
14 «L'aspect matériel du lieu n'est là que pour symboliser la clôture nécessaire à un état mental », écrit Hélène Cussac. «Les lieux de retraite dans quelques romans du XVIII siècle: d'une géographie réelle à la remise en cause d'un espace utopique ", dans Geographiae Imaginariae. Dresser le cadastre des mondes inconnus dans la fiction narrative de l'Ancien Régime, dir. Marie-Christine Pioffet, Paris, Hermann, 2014 [1éd. 2011], p. 137-158.

15 Saint François de Sales, Introduction à la vie dévote, I, III, dans Euvres, éd. André Ravier, «Bibliothèque de la Pléiade », Paris, Gallimard, 1969, p. 36.

16 Preuve que la femme n'existe pas socialement par elle-même, mais à travers son état. Voir Marcel Bernos, Femmes et gens d'Église dans la France classique. XVII'-XVIII siècle, Paris, Cerf, 2003, p. 39.
} 
L'importance des devoirs d'état est rappelée dans La Farfalla: ceux-ci «doivent tenir le premier rang après la loi générale, et passer avant certaines pratiques de dévotion ${ }^{17} »$. Marin ne manque pas, suivant en cela une tradition littéraire ancienne, de peindre les ridicules des fausses dévotes, et réaffirme la compatibilité entre la vie dévote et l'ordre social: "la véritable dévotion ne déplace rien » ( $V$., I, p. 23). Il tente ainsi d'accorder la radicalité du discours évangélique et la mesure de la vie sociale, rejoignant un idéal de modération qui est propre à la culture chrétienne classique. Il répond aux attaques dont la vie conventuelle est l'objet, de toutes parts, notamment au sein du clergé séculier, en opposant à ses contradicteurs non des arguments, mais des faits, par les vies de ses héroïnes dont l'exemplarité vertueuse doit s'imposer au lecteur. Il va jusqu'à figurer les relâchements dont une communauté s'était rendu coupable, pour mieux les opposer à la règle scrupuleusement suivie par une maison voisine, et donner enfin l'édifiant spectacle d'un couvent qui, une fois réformé, a atteint la perfection (An., I, p. 117 et suiv.). A la fois concession aux critiques qui dénoncent la corruption du modèle monastique ${ }^{18}$, et plaidoyer en faveur des couvents, cet exemple montre que Marin est conscient de l'incompréhension grandissante qui éloigne la société séculière de la culture religieuse, attentif à l'opinion et soucieux de lui donner une réplique adaptée, toujours par le biais de la fiction.

La figure de la veuve, très présente dans le roman de Marin, occupe dans la société du temps une place ambiguë, oscillant entre scandale et édification. Elle pose le problème de l'émancipation de la femme, soustraite par son mariage à l'autorité de son mari, et par son veuvage à celle de son époux ${ }^{19}$. Au cloître, où elle trouve bien souvent refuge, Marin préfère une autre solution, la vie séculière retirée. Il ouvre ainsi l'éventail des choix, prouvant au passage que l'Eglise évolue dans ses discours et dans ses pratiques. Il confirme enfin l'utilité sociale de la religion.

C'est en priorité aux jeunes filles que Marin s'adresse dans ses romans. Il conte les destins de vierges qui s'engagent, sous l'œil

\footnotetext{
${ }_{17}$ Marin, La Farfalla, ou la Comédienne convertie, Avignon, Vve Niel, 1762, p. 130.

${ }^{18}$ L'article de l'Encyclopédie « Ordre religieux » insiste sur le déclin des ordres dès l'origine du monachisme.

${ }^{19}$ Scarlett Beauvalet-Boutouyrie, Etre veuve sous l'Ancien Régime, Paris, Belin, 2001.
} 
attentif d'une maitresse avisée, dans la voie de la vie religieuse régulière. Loin de protester contre la violence qu'on leur ferait subir, comme la sœur Sainte-Suzanne, personnage de La Religieuse, elles chérissent leur état, et bénissent chaque jour le ciel d'avoir favorisé leurs vœux. Les parents ne poussent pas leurs filles vers le couvent, ils les en dissuaderaient plutôt. Virginie choque sa mère quand elle paraît devant elle revêtue d'une robe brune, aveu public de sa conversion. Comme une autre Marie-Madeleine, Marie Caraccioli veut embrasser une "vie de retraite et de pénitence ", animée qu'elle est d'un "désir qu'elle sentait dans son cœur d'y passer [dans le monastère] le reste de ses jours dans un oubli entier du monde, à pleurer ses péchés, et à s'immoler amoureusement à Jésus-Christ» ( $V$., I, p. 310).

\section{Le retrait hors de la clôture}

La voie d'une «profane séculière » n'est cependant pas moins difficile que celle d'une religieuse. Elle l'est davantage en réalité. Virginie ne s’y engage qu'à regret; malgré son extrême désir de la vie conventuelle, elle restera hors de la clôture. Ainsi en a décidé sa directrice. Sa vocation à elle est celle d'une « fille de renoncement et de patience», d'une "fille de croix». Elle vivra, à un degré paroxystique, la contradiction de la vie chrétienne ordinaire, le déchirement de la créature entre ce qu'elle doit à la société et ce qu'elle doit à Dieu. On retrouve le paradoxe, paradigme de toute existence, mais exprimé sur un mode volontaire : «je veux y être comme n'en étant pas » ( $V$., I, p. 275), déclare Virginie en écho à l'expression paulinienne «user de ce monde comme n'en usant point» (1 Co 7, 31). La tension n'est pas subie, elle est consentie. En cela il y a en effet vocation. Mère Scholastique précise les devoirs de la "vierge chrétienne »: " elle doit vivre retirée, fuyant de connaitre le monde et d'en être connue ; évitant les foules et préférant autant qu'il est à son pouvoir, d'être seule, plutôt que de se trouver avec les créatures » ( $V$., I, p. 379).

Pour décider d'une vocation, un long processus est nécessaire, qui inclut plusieurs retraites spirituelles au monastère. Ces retraites, de un à dix jours, comprennent des exercices spirituels, d'inspiration ignatienne, par exemple ceux que Marin préconise en appendice d'Adélaïde, ou dans sa Retraite spirituelle pour un jour de 
chaque mois (Avignon, Niel, 1763). Il est constamment rappelé que les pratiques de dévotion tendent à la vie cachée. Elles s'accordent aux temps du calendrier liturgique: l'Avent est une période de silence "pour imiter la vie cachée et silencieuse de Jésus-Christ dans le sein de sa mère » (V., I, p. 302); Virginie « se tenait plus retirée dans le Carême que dans les autres temps, pour tenir, disaitelle, compagnie à Jésus-Christ dans le désert » (ibid.). Contre une nature qui «tend toujours en bas» $(A d$., p. 201), la retraite a pour effet de fortifier l'homme intérieur, et d'engager une transformation morale et spirituelle radicale: «il faut que dans cette retraite vous soyez changées en nouvelles créatures » $(V$., I, p. 110 ${ }^{20}$. L'impudique devient chaste; le désordonné devient tempérant ; le faible et timide fort et courageux. Les valeurs que le monde tient en estime, il faut s'en déprendre, et tendre à l'opposé, notamment sur le point de la retraite: "Le monde aime à se produire, il faut aimer la retraite et la vie cachée » ( $V$., I, p. 138). «C'est là renoncer véritablement à soi-même que devenir tout autre que l'on était", écrit le père Rodriguez en commentant le verset Mt 16, 24 déjà citée ${ }^{21}$. Au terme de cette métamorphose, le retraitant est libéré des servitudes du monde. A partir de là rien ne s'oppose plus à la décision qu'il doit prendre sur l'état à choisir. La résolution d'Angélique advient au terme d'une grande retraite: "je veux penser plus sérieusement à changer de vie », confie-t-elle à la sœur Sophie Cunégonde (An., I, p. 378). Elle amène la postulante à rompre définitivement avec son ancienne vie, pour enfin trouver son vrai «centre». Le mot revient à plusieurs reprises dans la bouche ou sous la plume des vierges de Marin. Il traduit la certitude radieuse d'être à sa place. "Mon cœur s'y [dans cette pensée] repose comme dans son centre ; tout m'y plaît aussi, et tout m'attire à la religion », avoue Rosalie ( $V$., I, p. 188).

\section{L'ermite au féminin}

Le $17^{\mathrm{e}}$ siècle a cru voir se renouveler les merveilles des vies d'anachorètes des déserts de l'Égypte ancienne. Jeanne-Marguerite,

\footnotetext{
20 Voir 2 Co 4, 16 («Mais encore que dans nous l'homme extérieur se détruise, néanmoins l'homme intérieur se renouvelle de jour en jour »).

21 Alphonse Rodriguez, Pratique de la perfection chrétienne, Toulouse, 1747, t. II, p. 346.
} 
la Solitaire des Rochers, a vécu dans les dernières années du Grand Siècle une vie érémitique d'un total dénuement. Les lettres échangées avec son directeur, le père Luc de Bray, et qui circulèrent dès son vivant, attestent la vérité des faits. L'histoire qui en est tirée, suivie de la correspondance, paraît en $1787^{22}$. Cette vie au désert, dans les montagnes des Pyrénées, d'abord dans une grotte, puis dans un vallon infesté de serpents, est digne de sainte Euphrasie, qui, pour vaincre les tentations, ne prit aucune nourriture pendant quarante jours, ou de sainte Alexandra, qui passa dix ans recluse dans un tombeau. De cette histoire ou d'autres semblables, Marin tire l'idée d'un ermite féminin de la région de Montréal en Sicile. Marie de Libératis, devenue la Solitaire de la Madona Sanctissima, si elle dépend d'un couvent de clarisses, où elle a été novice et dont elle porte l'habit, vit parfaitement seule dans la montagne, dans un ermitage que sa famille lui a fait construire. Ce mode de vie, qui fait suite à un vœu, est présenté comme extraordinaire chez une fille. Il s'autorise des premiers temps du monachisme: "[Les anciens solitaires] s'éloignaient absolument de leur pays, afin d'être à Dieu sans obstacle; et ils ne connaissaient d'autre patrie que la céleste Jérusalem », explique Marie à sa mère ( $V$., II, p. 209-210). Après avoir découvert trois grottes dans la montagne, la jeune fille s'y retire "pour mieux seconder l'attrait que le Seigneur lui avait donné pour la retraite et la vie cachée»( $V$., II, p. 257). Elle complète plus tard son installation par un calvaire. Les prolongements que Marin donne à cette histoire suggèrent pourtant que l'érémitisme n'est qu'une forme transitoire de vie retirée, et qu'il ne saurait exister de façon autonome. De fait, Marie est bientôt rejointe par deux autres solitaires, et, aidées d'une servante, toutes trois forment une petite communauté de filles pieuses. C'est le monachisme anachorétique, tel que le décrivent les historiens des premiers temps du christianisme : la vie collective y

${ }^{22}$ La Solitaire des Rochers, Chateaufort, 1787. - Pierre Naudin, ouvr. cité, p. 113-115. Voir Dinah Ribard, "Radicales séparations », Archives de sciences sociales des religions, $\mathrm{n}^{\circ} 150$, Paris, EHESS, 2010, p. 117-133. 
est réduite à la portion congrue, l'essentiel de l'existence se passant dans l'isolement et le silence ${ }^{23}$.

Autre anachorète moderne, non plus sicilien mais avignonnais, Anne Violet, qui après s'être vu refuser l'état monastique, se résolut à vivre entre ses parents, puis seule après leur mort, une existence retirée, pauvre et laborieuse, et cependant réglée comme en un couvent. Marin, qui en conte l'histoire, qu'il fait suivre des Mémoires de l'intéressée, la présente comme véridique, l'assortissant de toutes sortes de détails à fonction crédibilisante. Cette religieuse domestique, tierçaire franciscaine, vit dans sa maison comme en un "retranchement", et ne se distingue par rien de singulier. "On ne remarquait point qu'elle affectât dans ses entretiens une vertu farouche et concentrée, qui la rendît singulière ou difficile ${ }^{24} »$. Son goût extrême pour les macérations, les aliments gâtés, l'épuisement physique dans le travail, la modestie de son logis, qu'« on ne peut guère mieux comparer qu'à ces pauvres chaumières des anciens ermites de la Thébaïde ${ }^{25}$ ", tout enfin signale l'héroïque sainteté. Ce détachement radical lui vaut des lumières surnaturelles, un don visionnaire et prophétique, une éloquence persuasive.

Cette fascination qu'exerce sur Marin la figure du solitaire, qui s'explique aussi par l'ordre auquel il appartient, et dont le fondateur, le calabrais François de Paule, commença par être ermite, suggère à quel point la notion de " dévotion aisée » lui est étrangère. "On n'entre au ciel qu'en se faisant violence », disait le patriarche des Indes à la marquise de Los Valientes ${ }^{26}$. La voie est non seulement étroite, mais encore semée de croix. La dévotion qui prétend se concilier les joies du monde est "chimérique», «monstrueuse » $(V \text {., I, p. 135 })^{27}$.

\footnotetext{
23 Bernard Flusin, "L'essor du monachisme oriental », dans Histoire du christianisme des origines à nos jours, t. III, Paris, Desclée, 1998, p. 545-608.

${ }^{24}$ Marin, Conduite spirituelle de la Scur Anne Violet, du Tiers-Ordre de S. François d'Assise, Avignon, Giroud, 1740, p. 22.

${ }^{25}$ Id., p. 91.

${ }_{26}$ Marin, La Marquise de Los V alientes, ou la Dame chrétienne. Histoire castillane, Avignon, Niel, 1765, II, p. 28.

${ }^{27}$ Marin s'écarte du point de vue exprimé par le R.P. Pierre Le Moyne dans La dévotion aisée (1652), déjà vivement attaqué par Pascal dans Les Provinciales (9e Lettre). Voir MarieMadeleine Fragonard, «Déserts romanesques et dévotion aisée », dans Le Désert, l'espace et
} 
Cependant lors même que Marin insiste sur la radicalité de la retraite, condition nécessaire mais non suffisante du salut, il en privilégie la valeur exemplaire et les conséquences sociales. Virginie, touchée par l'exemple de Marie de Libératis, veut l'imiter : «On eût dit qu'elle voulait vivre de la vie des anciens ermites » $(V$., II, p. 194). Cette imitation en chaîne produit sur la population entière une impression édifiante. Anne Violet, par son action charitable durant la grande peste, sa sollicitude pour ses parents, sa vie de pauvre parmi les pauvres, est une figure davantage acceptable pour un $18^{\mathrm{e}}$ siècle qui n'admet d'autre vertu que sociale. $\mathrm{Ni}$ religieuse, ni ermite, ni fondatrice d'ordre, son engagement au service de ses semblables fait d'elle une bienfaitrice de l'humanité, et pour l'incroyant une sainte laïque.

\section{Ni monde, ni cloître : le troisième lieu}

Marin introduit, et c'est l'originalité de ses romans, une voie médiane entre le monde et le cloître, offrant aux jeunes filles la possibilité de vivre leur état de recluses sans quitter formellement le monde. Le modèle, s'il s'inspire d'ermites féminins du TiersOrdre franciscain, est une authentique création de l'auteur. Il porte sa marque. Aussi ne le définit-il que par comparaison contrastive avec d'autres vocations, au premier rang desquelles la vie conventuelle. Mère Scholastique comprend, «en comparant les dispositions de sa nièce avec celles de Rosalie, que leurs voies seraient différentes, ainsi que leur vocation » (V., I, p. 115). D'un côté la voie régulière, de l'autre la voie séculière. Le diptyque a l'avantage de faire sentir ce qui différencie les deux voies, tout en marquant les points de convergence. Car la vierge n'est pas moins recluse que la religieuse : elle l'est différemment. Le périmètre de sa clôture, dans la mesure où il n'est pas défini par une quelconque autorité institutionnelle, est à réinventer tous les jours. La retraite volontaire n'est-elle pas la meilleure réponse à faire aux critiques que les philosophes adressent au système conventuel, et à la contrainte qu'il exercerait sur les esprits? En promouvant une forme d'état qui ne suppose de la part de celui qui s'y engage

l'esprit (Moyen Age-XXe siècle), dir. Frank Lestringant et Sarga Moussa, Revue des Sciences Humaines, $\mathrm{n}^{\circ} 258,2000$, p. 163-176. 
aucun vœu formel, et qui à ce titre le laisse libre juridiquement de mener la vie qu'il souhaite, Marin détruit le spectre de la vocation forcée, et réhabilite le modèle monastique, qui est selon lui le miroir concentrique du christianisme.

Pour autant, l'auteur ne perd pas de vue son objectif: convertir le monde en substituant la loi évangélique à la loi humaine. Or quelle société mieux que la communauté religieuse applique à la lettre les préceptes de l'évangile ? Il s'agira donc d'imaginer une transplantation de la règle monastique à la vie civile; et c'est, dans le roman de Marin, à une veuve qu'on doit cette adaptation expérimentale. À la mort de son mari, Sophie de Casa-Sancta, la bien nommée, transforme sa demeure en «une espèce de monastère » $(V$., I, p. 117), et $\mathrm{y}$ vit avec ses filles selon une règle inspirée de saint Benoit. D'autres familles veulent l'imiter. Le bourg entier, opportunément nommé Gli-Angeli, compte bientôt autant de monastères que de maisons. Revient ici en mémoire le souvenir de la cité antique d'Oxirinque, en BasseThébaïde, dont l'abbé Fleury donne, dans son Histoire ecclésiastique, une description détaillée ${ }^{28}$. Cette ville, qui se résumait à «un seul monastère ", et ne comptait pas un seul païen, ni un seul hérétique, toujours selon Fleury, est l'exacte antithèse de La République des incrédules, dialogue antiphilosophique où est débattue la question de savoir si une société d'athées vertueux est viable ${ }^{29}$.

La retraite, dans le roman profane, advient souvent au terme d'une vie de tribulations; elle est aussi le prétexte d'un récit rétrospectif qui doit éclairer les raisons de la sécession volontaire. Il n'en est pas de même chez Marin. La retraite y est bien plutôt un point de départ, à partir duquel peut se déployer la vie de l'esprit; elle est la condition de possibilité de toute vie chrétienne. La radicalité de la séparation, qu'elle soit physique ou mentale, fait comprendre que le roman de Marin ne fait pas seulement l'apologie des monastères contre ceux qui comme Mercier y voient

${ }^{28}$ Claude Fleury, Histoire ecclésiastique, t. V, Paris, Desaint et Saillant, 1750, p. 26. Repris par Marin dans Vies des Pères des déserts d'Orient, Avignon, Vve Niel, 1761, I, p. 102-104.

${ }^{29}$ Marin, Le Baron van Hesden, ou la République des incrédules, Toulouse, Birosse, 1762. Voir

Raymond Trousson, "Michel-Ange Marin et les Pensées philosophiques», Recherches sur Diderot et sur l'Encyclopédie, n 13, octobre 1992, p. 47-55. 
les «déplorables monuments d'une antique superstition ${ }^{30}$ ", mais aussi, et peut-être surtout contre les tenants d'une dévotion accommodée au siècle.

Le propos n'échappe pourtant pas au reproche d'ambiguité qu'on pourra lui adresser. Voie universelle ou voie d'élection ? Vie dans le monde ou contre le monde? Dévotion ordinaire ou dévotion héroïque ? L'ermite, dont Marin projette la silhouette à l'arrière-plan de la vie retirée, a certes une odeur suave, comme chacun sait; il a aussi un parfum de scandale. Il est, a fortiori quand il se décline au féminin, l'emblème d'une religion en rupture avec la société. Les vierges sages de Marin, dont les voies s'accordent si merveilleusement avec l'ordre des familles et les lois des hommes, se rêvent, à l'exemple des anciens anachorètes, en sauvages de la religion.

Malgré ces contradictions, ou à cause d'elles, Marin réussit ce tour de force de rendre la vie dévote intéressante, sans recourir aux facilités du merveilleux ni aux excès du mysticisme. S'en tenant aux ressources minimales offertes par le genre romanesque, il parvient à raconter un non-événement, à représenter l'irreprésentable intériorité, à faire un spectacle de ce qui cherche à se dérober aux yeux du monde. En combinant la narration et le discours, le portrait et la prescription, en faisant dialoguer ses personnages ou en les faisant correspondre, il ouvre un accès à l'intelligence de la vie cloîtrée, à sa subtilité, à sa fragilité, à ses paradoxes ; et parvient à communiquer à ses lectrices un réel attrait pour la retraite. Enfin, en reconnaissant à la femme une prérogative à la vie retirée, l'auteur déclare sa foi en sa fécondité spirituelle. Contre Usbek, qui décrivait chaque maison religieuse comme «une famille éternelle, où il ne naît personne", et les couvents comme "autant de gouffres, où s'ensevelissent les races futures ${ }^{31} »$, Marin pense que l'avenir appartient aux solitudes virginales, et que de là doit naître l'humanité nouvelle. De la lutte que se livrent clercs et philosophes pour capter le plus large public en usant de formes littéraires séduisantes, Marin ne sort-il pas gagnant? Les rééditions de

30 Louis-Sébastien Mercier, Tableau de Paris, VII, "Couvents. Religieuses », éd. JeanClaude Bonnet, Paris, Mercure de France, 1994, t. II, p. 77.

${ }^{31}$ Montesquieu, Lettres persanes, lettre 113, dans CEuvres, t. I, éd. Jean Ehrard et Catherine Volpilhac-Auger, Voltaire Foundation, Oxford, 2004, p. 448. 
Virginie ou d'Adélaïde de Witsbury sont légions, au $18^{\mathrm{e}}$ siècle, ce dont témoigne Chateaubriand qui en 1803 dit qu'elles sont «fort répandues $^{32} »$, et plus encore au $19^{\mathrm{e}}$ siècle, accompagnant la restauration des congrégations religieuses et contribuant à diffuser, par ces histoires de vocations juvéniles, le thème des anges sur la terre. Thérèse de Lisieux, en sa "petite voie », n'est-elle pas une fidèle héritière des héroïnes de Marin ?

Nicolas BRUCKER

Université de Lorraine

32 Chateaubriand, ouvr. cité, ibid. - Ni Philippe Martin, qui qualifie Virginie de «bestseller» (Une religion des livres (1640-1850), Paris, Cerf, 2003, p. 72), ni Francis Marcoin, qui indique que les œuvres de Marin «seront abondamment rééditées jusqu'à la fin du XIX siècle » (Librairie de jeunesse et littérature industrielle an XIX e siècle, Paris, Honoré Champion, 2006, p. 217) ne fournissent le moindre chiffre. 